\title{
Influence of gaseous hydrogen on plastic strain in vicinity of fatigue crack tip in Armco pure iron
}

\author{
Tomoki Shinko ${ }^{1}$, Gilbert Hénaff ${ }^{1, *}$, Damien Halm $^{1}$ and Guillaume Benoit ${ }^{1}$ \\ ${ }^{1}$ Pprime Institute, Department of physics and mechanics of materials, ISAE-ENSMA, 86961 Futuroscope Chasseneuil Cedex, France
}

\begin{abstract}
A multi-scale characterisation of the crack tip plasticity has been investigated in a fatigue crack propagation under gaseous hydrogen at gas pressure of $35 \mathrm{MPa}$ in a commercially pure iron, Armco iron. The dislocation structure beneath a fracture surface was observed by a Transmission Electron Microscopy (TEM), and the cyclic and monotonic plastic zones were evaluated by an Out-of-Plane Displacement (OPD) measurement. By the TEM observation in a non-accelerated regime $\left(\Delta K=11 \mathrm{MPa} \times \mathrm{m}^{1 / 2}\right)$, a dislocation cell structure was observed even in the brittle intergranular fracture in hydrogen. This result indicates a certain amount of plastic strain is introduced into the grains in front of an intergranular crack in hydrogen, and this may explain the mechanism of hydrogen-induced intergranular fatigue crack propagation. On the other hand, in an accelerated regime $\left(\Delta K=18\right.$ and $20 \mathrm{MPa} \times \mathrm{m}^{1 / 2}$ ), a distribution of scattered dislocation tangles without any cell or vein structure was observed in hydrogen. Besides, the inhibition of the cyclic plasticity near the crack path in hydrogen was confirmed by the OPD measurement. These results are clear evidences of hydrogen-induced localization of cyclic plasticity in the vicinity of a crack tip, which suggests a mechanism model of hydrogen-enhanced fatigue crack growth based on the plasticity localization.
\end{abstract}

\section{Introduction}

Hydrogen is expected as one of the most desired energy carriers in the future infrastructures of a sustainable society. On the other hand, it is known that gaseous hydrogen environment may result in a significant degradation of mechanical properties in metallic materials which is commonly known as "hydrogen embrittlement" [1-3]. Especially, the degradation of fatigue resistance due to hydrogen environment is a crucial problem for the industrial usage. Many theories have been proposed to explain the mechanism of hydrogen embrittlement. In particular, the following three theories are today considered as the most important ones: Hydrogen-Enhanced DEcohesion (HEDE) [4], Hydrogen-Enhanced Localized Plasticity (HELP) [5] and Adsorption-Induced Dislocation Emission (AIDE) [6]. The HEDE mechanism supposes that the hydrogen atoms adsorbed at a crack tip or segregated at the grain boundaries weaken interatomic bonds and cause an atomic decohesion. This leads to a brittle fracture such as a cleavage fracture and an intergranular decohesion. In the HELP mechanism, the solute hydrogen atoms in front of the crack tip enhance and localize a dislocation activity. This promotes a nucleation and coalescence of micro voids, resulting in a crack propagation with less blunting and less macroscopic ductility of the material. The fracture surface often exhibits small, shallow dimples. The AIDE mechanism is based on the facilitation of dislocation nucleation due to adsorbed hydrogen. In this mechanism, adsorbed hydrogen on the surface and the sub-surface weakens interatomic bonds and promotes the dislocation nucleation at a crack tip which enhances the local plastic strain near the crack tip. The principle of FCG enhancement by the AIDE is similar with the HELP mechanism, however the main difference is that the AIDE involves the sub-surface effect of adsorbed hydrogen while the HELP is based on the solute hydrogen in the material.

In the previous researches by Pprime institute $[7,8]$, it has been confirmed that the Fatigue Crack Growth Rates (FCGRs) in Armco iron under $35 \mathrm{MPa}$ of gaseous hydrogen environment are accelerated up to 100 times higher than the one in air, as shown in Fig. 1. And also, the FCGRs in hydrogen exhibit different tendencies depending on the stress intensity factor range $\Delta \mathrm{K}$. These tendencies can be decomposed into following three distinct regimes: (a) a non-accelerated regime $(\Delta K<12$ $\mathrm{MPa} \times \mathrm{m}^{1 / 2}$ ) where FCGR is not accelerated and is almost the same as in air; (b) a transition regime $(12<\Delta K<14$ $\mathrm{MPa} \times \mathrm{m}^{1 / 2}$ ) where FCGR is sharply accelerated; and (c) an accelerated regime $\left(14 \mathrm{MPa} \times \mathrm{m}^{1 / 2}<\Delta K\right)$ where the slope of FCGR becomes the same level as in air. It has also been confirmed that the hydrogen-induced FCG enhancement depends on the loading frequency $f$. As well as the FCGR is affected by hydrogen, the fracture mode is also clearly changed due to hydrogen. In the non-accelerated regime, the fracture mode is brittle intergranular fracture in hydrogen while a ductile transgranular quasi-cleavage (QC) fracture takes place in air. In the transition regime, fracture mode in hydrogen

Corresponding author: gilbert.henaff@ensma.fr 
gradually changes to a transgranular QC fracture. Subsequently in the accelerated regime, the fracture surface becomes fully transgranular QC fracture with course and flat striations (i.e. brittle striations).

In this study, in order to clarify the mechanism of the hydrogen-induced FCG enhancement in Armco iron, the influence of hydrogen on microscopic and macroscopic plasticity at a crack tip has been investigated by means of the following two methods: (1) a microscopic plasticity near the crack tip has been investigated by a Transmission Electron Microscopy (TEM) of dislocation structure immediate beneath fracture surface; and (2) macroscopic cyclic and monotonic plasticity in front of the crack tip has been evaluated by a measurement of lateral shrinkage around the fatigue crack path (i.e., Out-of-Plane Displacement, OPD) by using an interferometric confocal microscope.

\section{Material and testing method}

The material used in this study is a commercially pure iron, Armco iron (AK Steel Corp.). The chemical composition of Armco iron is shown in Table 1. An annealing heat treatment at $1073 \mathrm{~K}\left(800{ }^{\circ} \mathrm{C}\right)$ for 60 minutes was applied in order to relax a residual stress and homogenize the ferrite microstructure. Figure 1 shows an Electron Back-Scattered Diffraction (EBSD) mapping of the heat-treated Armco iron. The mean grain size after heat treatment is approximately $90 \mu \mathrm{m}$. The mechanical properties determined after heat treatment are: yield strength $\sigma_{y}=170 \mathrm{MPa}$ and maximum elongation $A=25 \%$.

Specimen preparation and fatigue crack propagation tests were performed by following the ASTM E647 standard. CT specimens with a width $\mathrm{W}=$ $40 \mathrm{~mm}$ and a thickness $\mathrm{B}=8 \mathrm{~mm}$ were machined in the longitudinal-transverse orientation against hot-rolling direction. The specimen surface was finished by diamond abrasives with a diameter of $1 \mu \mathrm{m}$.

Fatigue crack propagation tests were conducted under loading control at room temperature under various atmospheres: vacuum $\left(5 \times 10^{-10} \mathrm{~Pa}\right)$, laboratory air, 3.5

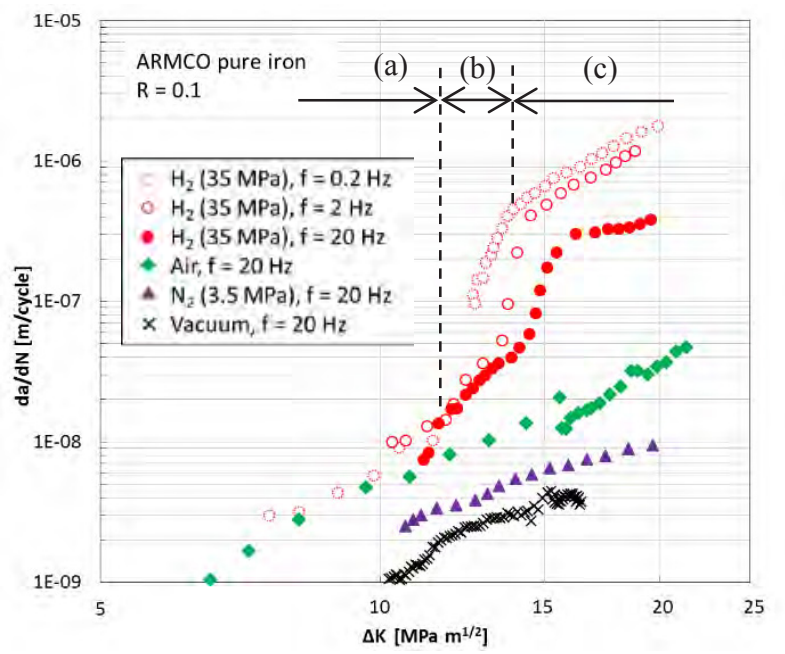

Fig. 1. FCGRs $(=\mathrm{da} / \mathrm{dN})$ vs $\Delta \mathrm{K}$ in Armco iron [8]. FCGR curves in hydrogen can be separated as three distinct regime: (a) a non-accelerated regime, (b) a transition regime and (c) an accelerated regime.
$\mathrm{MPa}$ of gaseous nitrogen and $35 \mathrm{MPa}$ of gaseous hydrogen. The tests were performed by using Hycomat test bench [9] developed by Pprime institute. It consists of an autoclave assembled to a servo-hydraulic machine, which has a capacity to perform mechanical tests under a maximum gas pressure of $40 \mathrm{MPa}$ at the highest temperature of $150{ }^{\circ} \mathrm{C}(423 \mathrm{~K})$. The various loading frequencies of $0.2,2$ and $20 \mathrm{~Hz}$ were applied to investigate its influence on FCGR. Crack length was measured by optical microscope and a Direct Current Potential Drop (DCPD) technique during the tests. The stress ratio $R\left(=K_{\min } / K_{\max }\right)$ was 0.1 , where $K_{\min }$ and $K_{\max }$ are the minimum and maximum stress intensity factors, respectively.

\section{Result}

\subsection{TEM observation of dislocation structure immediately beneath fracture surface}

To investigate the microscopic plasticity immediate vicinity of a crack tip, the dislocation structure in the material within $10 \mu \mathrm{m}$ depth from fracture surface has been observed by TEM. The thin foils for TEM observation were prepared from the fracture surfaces by using a Focused Ion Beam (FIB, Helios NanoLab G3, FEI Company). The thickness of foil was approximately $100 \mathrm{~nm}$. Each TEM foil presented in this study consists of one grain, as confirmed by a transmission EBSD analysis on the foils. Figure 3 shows the SEM images of fracture surfaces and the dark-field TEM images of cross-section immediately beneath the fracture surface at $\Delta K=11 \mathrm{MPa} \times \mathrm{m}^{1 / 2}$ at $f=$ $20 \mathrm{~Hz}$ in air (Fig. 3 (a)) and at $f=2 \mathrm{~Hz}$ in $35 \mathrm{MPa}$ of gaseous hydrogen (Fig. 3 (b)). This $\Delta K$ value corresponds to the non-accelerated regime. A rectangular part with different contrast in the center of some SEM

Table 1. Chemical composition of Armco iron (mass \%).

\begin{tabular}{c|ccccccc}
\hline Element & $\mathrm{C}$ & $\mathrm{Mn}$ & $\mathrm{P}$ & $\mathrm{S}$ & $\mathrm{Cu}$ & $\mathrm{N}$ & $\mathrm{Al}$ \\
\hline Content & 0.001 & 0.050 & 0.003 & 0.003 & 0.009 & 0.004 & 0.005 \\
\hline Element & $\mathrm{Cr}$ & $\mathrm{Mo}$ & $\mathrm{Ni}$ & $\mathrm{Sn}$ & $\mathrm{Si}$ & \multicolumn{2}{c}{$\mathrm{Fe}$} \\
\hline Content & 0.015 & 0.002 & 0.14 & 0.002 & 0.004 & \multicolumn{2}{c}{ Balance } \\
\hline
\end{tabular}

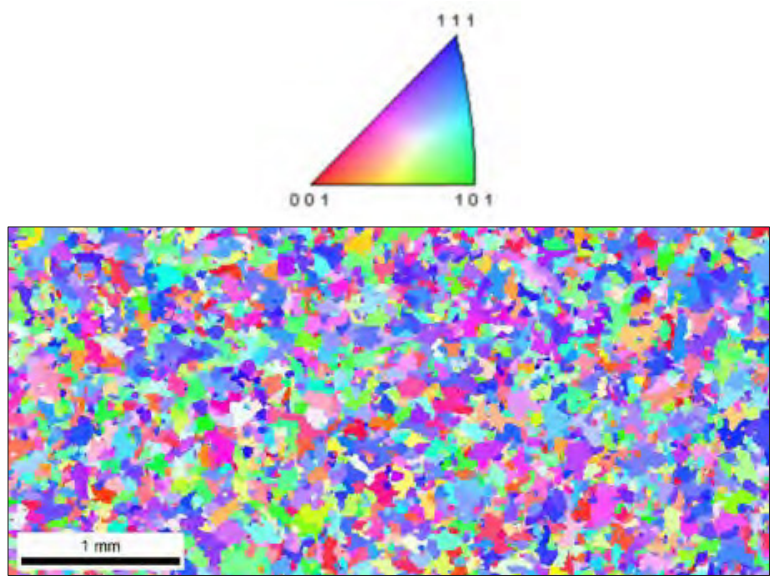

Fig. 2. EBSD mapping of microstructure of ARMCO iron used in this study. 
images is an area covered by platinum layer to protect the material from the FIB milling.

The fracture surface in air (Fig. 3 (a-1)) represented a typical transgranular QC fracture. This fracture mode in air continued until the unstable rupture at the end of the test. In the TEM image (Fig. 3 (a-2)), clear dislocation walls in the inside of material can be observed. Besides, it can be observed that the dislocation walls form a cell structure, in other words sub-grains, with a diameter of few $\mu \mathrm{m}$. On the other hand, one can see that the fracture surfaces in hydrogen (Fig. 3 (b-1) and (b-3)) are fully covered by intergranular facets evidencing an intergranular fracture. And also, some secondary intergranular cracks and stripe-like plastic markings on the surface of intergranular facets have been observed as shown in Fig. 3 (b-3) and (b-4). The presence of the plastic markings suggests that a certain amount of plastic strain is introduced to the grains in front of the crack tip. Both TEM image of the dislocation structure in hydrogen (Fig. 3 (b-2) and (b-5)) shows a similar dislocation cell structure as observed in air. The diameter of dislocation cells is approximately $1 \mu \mathrm{m}$ which is slightly smaller than the one in air.

Figure 4 shows the same type of SEM and TEM observations in case of $\Delta K=20 \mathrm{MPa} \times \mathrm{m}^{1 / 2}$ in the accelerated regime. It has been confirmed that the fracture surface showing the transgranular QC fracture (Fig. 4 (a-1)) and the dislocation structure showing the cell structure (Fig. 4 (a-2)) are not changed from the ones observed in non-accelerated regime (Fig. 3 (a)). On the other hand, as shown in Fig. 4 (b-1) and (b-4), the fracture surfaces at $\Delta \mathrm{K}=20 \mathrm{MPa} \times \mathrm{m}^{1 / 2}$ in hydrogen represented a transgranular QC fracture with brittle striations. Figure 4 (b-2) and (b-5) show the entire TEM images of the foils, and Fig. 4 (b-3) and (b-4) are the magnified images of the areas indicated by the rectangles

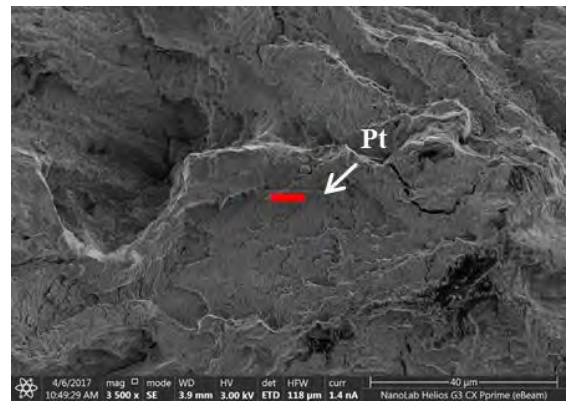

(a-1) SEM image of fracture surface.

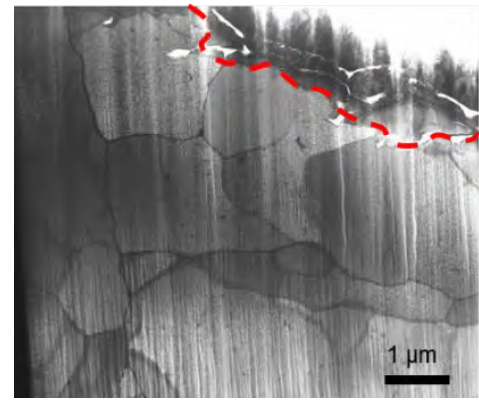

(a-2) TEM image of cross-section at the position indicated by the red line in (a-1).

(a) In air, $f=20 \mathrm{~Hz}$.

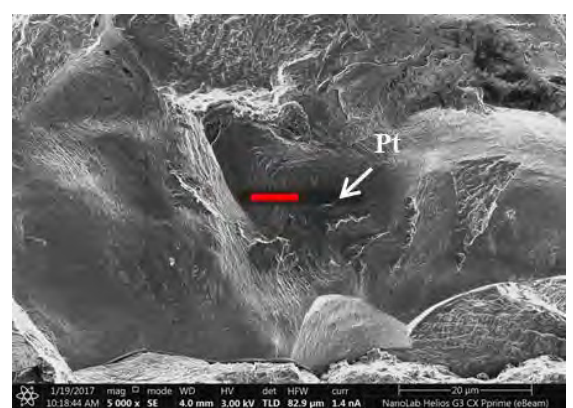

(b-1) SEM image of the first position of fracture surface.

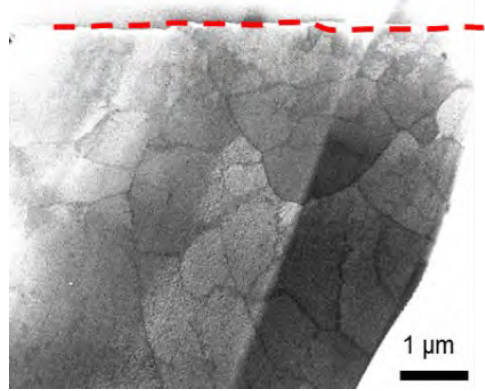

(b-2) TEM image of cross-section at the position indicated by the red line in $(b-1)$.

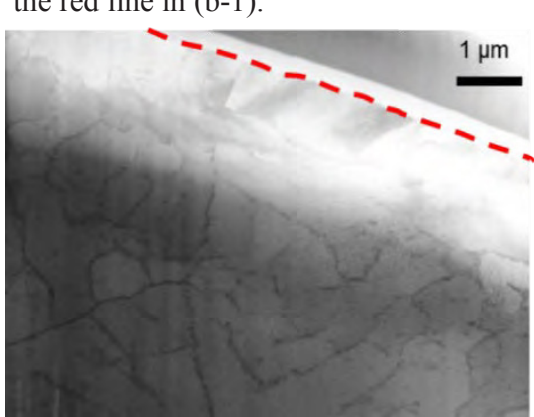

(b-5) TEM image of cross-section at the position indicated by the red line in (b-3). (b-4) Higher magnified SEM image of stripe-like plastic markings in the area indicated by the red rectangle in (b-3).

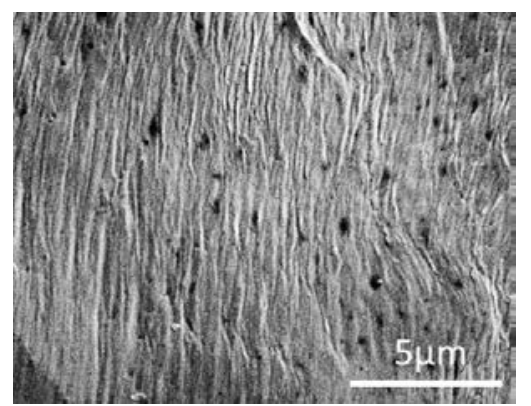

(b) In $35 \mathrm{MPa}$ of gaseous hydrogen, $f=2 \mathrm{~Hz}$.

Fig. 3. SEM images of fracture surfaces and TEM images of cross-section immediately beneath fracture surface at $\Delta K=11$ $\mathrm{MPa} \times \mathrm{m}^{1 / 2}$ in air, $f=20 \mathrm{~Hz}$ and in gaseous hydrogen $(35 \mathrm{MPa}), f=2 \mathrm{~Hz}$. Crack propagation direction is from left to right in the images. Red dotted lines in TEM images show the position of fracture surface. 
in Fig. 4 (b-2) and (b-5). The red arrows in the SEM and TEM images in hydrogen indicate the position of the striations crossing the TEM-observed area. In these TEM images in hydrogen, the dislocation walls do not form any cell or vein structure, but are disconnected in short pieces and randomly dispersed. In addition, one can notice that there are long dislocation walls starting from the positions of striations, more precisely, one starting from the striation in the center of Fig. 4 (b-2) and another one starting from the striation in the left side of Fig. 4 (b-5) and (b-6), even though there is no other long dislocation wall observed. The dislocation wall in Fig. 4 (b-2) is separated into two and winding, while another one in Fig. 4 (b-5) and (b-6) is straight and angled approximately 45 degrees from the fracture surface.

\subsection{Measurement of Out-of-Plane Displacement (OPD) along crack path}

In order to investigate the macroscopic plasticity (cyclic and monotonic plasticity) in vicinity of the crack tip, the OPD has been measured by using an interferometric confocal microscope (Talysurf CCI6000, Taylor Hobson).

The surface relief curves measured at $\Delta K=12$ and $18 \mathrm{MPa} \times \mathrm{m}^{1 / 2}$ in various testing conditions are shown in Fig. 5. By comparing Fig. 5 (a) and (b), one can confirm that the displacement and the width of OPD increase with the increment of $\Delta K$ from 12 to $18 \mathrm{MPa} \times \mathrm{m}^{1 / 2}$. The black dotted line shows the half of monotonic plastic zone size $r_{p} / 2$. The monotonic plastic zone size in plane

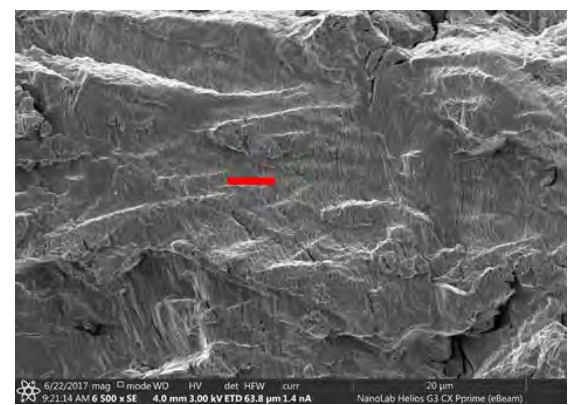

(a-1) SEM image of fracture surface.

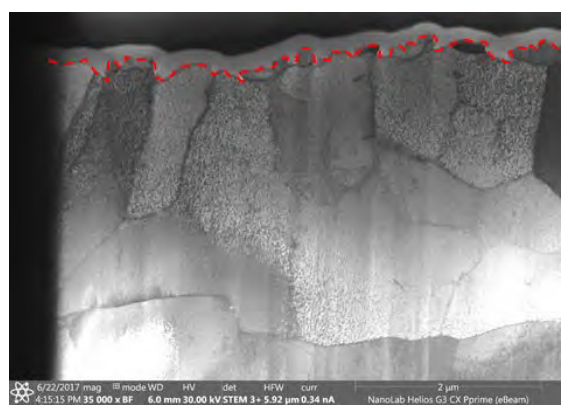

(a-2) TEM image of cross-section at the position indicated by the red line in (a-1).

(a) In air $(f=20 \mathrm{~Hz})$.

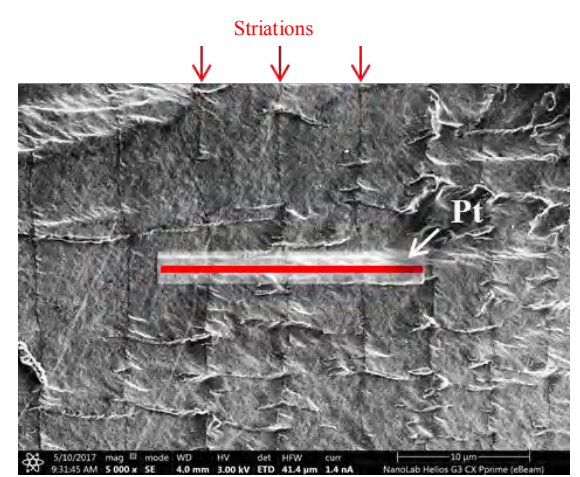

(b-1) SEM image of the first position of fracture surface. Striations $\downarrow \downarrow \downarrow \downarrow \downarrow$

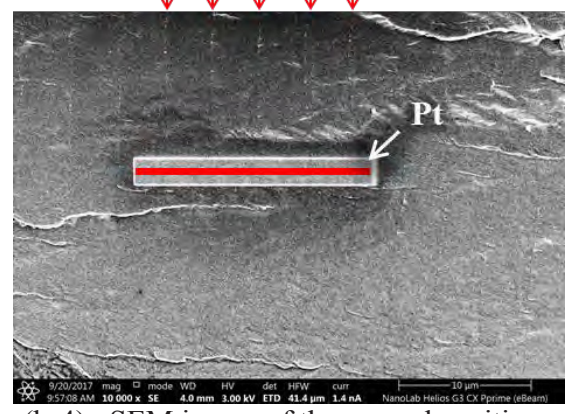

(b-4) SEM image of the second position of fracture surface.

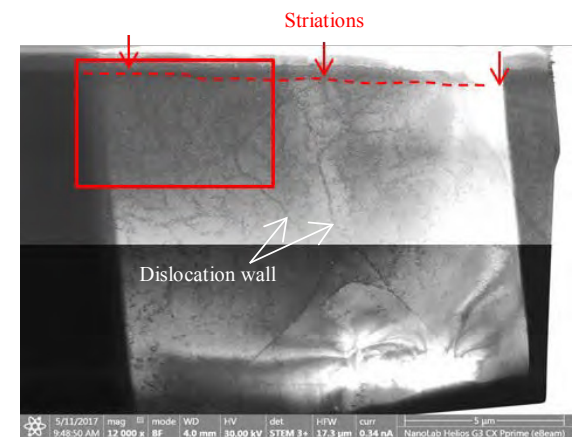

(b-2) TEM image of cross-section at the position indicated by the red line in (b-1).

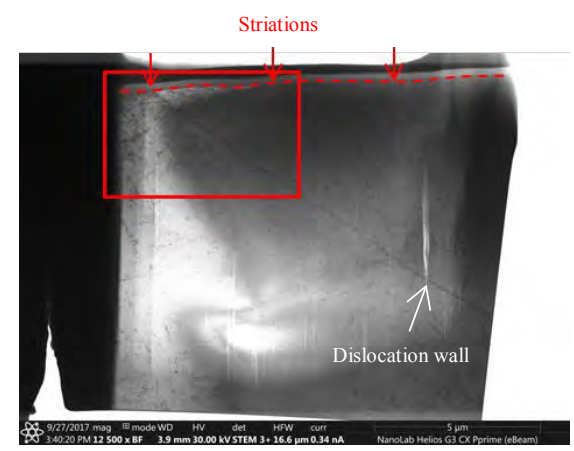

(b-5) TEM image of cross-section at the position indicated by the red line in (b-4).

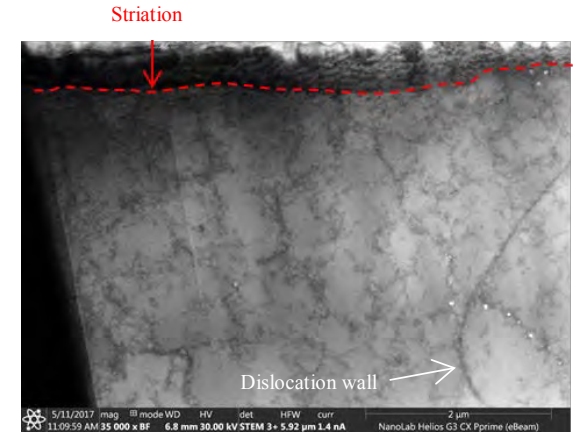

(b-3) Higher magnified TEM image of the area indicated by the red rectangle in $(b-2)$.

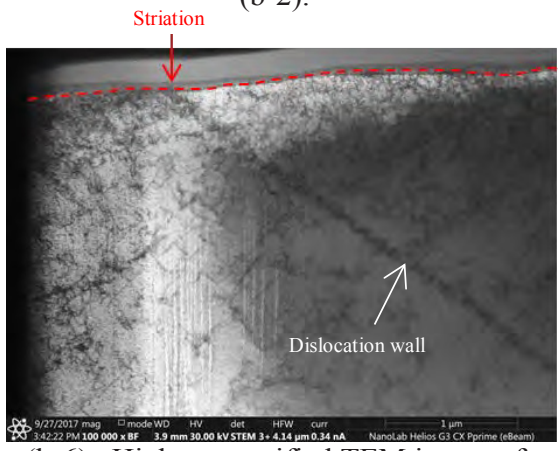

(b-6) Higher magnified TEM image of the area indicated by the red rectangle in (b-5).

(b) In $35 \mathrm{MPa}$ of gaseous hydrogen $(f=2 \mathrm{~Hz})$.

Fig. 4. SEM images of fracture surfaces and TEM images of cross-section immediately beneath fracture surface at $\Delta K=20$ $\mathrm{MPa} \times \mathrm{m}^{1 / 2}$ in air $(f=20 \mathrm{~Hz})$ and in $35 \mathrm{MPa}$ of gaseous hydrogen $(f=2 \mathrm{~Hz})$. Crack propagation direction is from left to right in all images. Red dotted lines show the location of fracture suface. Red arrows show the position of striations crossing the TEM-observed area. 
stress condition $r_{p}$ was calculated by the following equation [10]:

$r_{p}=\frac{1}{\pi}\left(\frac{K_{\max }}{\sigma_{y}}\right)^{2}$.

The values of $r_{p} / 2$ are obtained as $0.85 \mathrm{~mm}$ at $\Delta K=12$ $\mathrm{MPa} \times \mathrm{m}^{1 / 2}$ and $1.92 \mathrm{~mm}$ at $\Delta K=18 \mathrm{MPa} \times \mathrm{m}^{1 / 2}$, which are close to the positions of the beginning of OPD. Therefore, the observed OPD is considered as a result of monotonic plasticity around the crack tip. In a comparison of the surface relief curves, no clear influence of environment or testing conditions on the

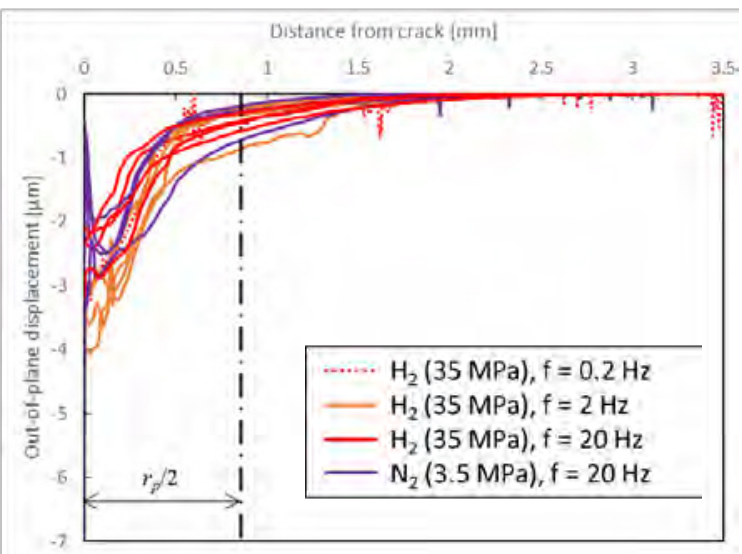

(a) $\Delta K=12 \mathrm{MPa} \times \mathrm{m}^{1 / 2}$
OPD was confirmed except for a quite large fluctuation in the surface relief curves of each specimen. This result denotes that hydrogen does not influence on the large scale of plastic strain in a monotonic plastic zone.

Figure 6 shows the surface topographic images of side-surface near the crack path at $\Delta K=12$ and 18 $\mathrm{MPa} \times \mathrm{m}^{1 / 2}$ in nitrogen and in hydrogen (at $f=20 \mathrm{~Hz}$ ). Color gradation indicates surface height. Central vertical

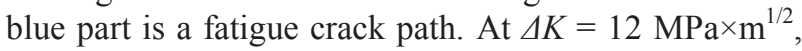
the surface topographies in both environments (Fig. 6 (a1) and (b-1)) are smooth. On the other hand, at $\Delta K=18$

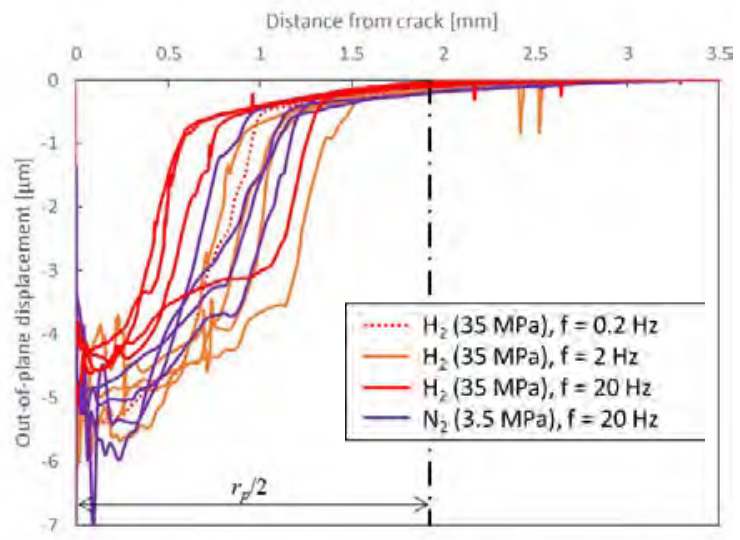

(b) $\Delta K=18 \mathrm{MPa} \times \mathrm{m}^{1 / 2}$

Fig. 5. Surface relief curves presenting the Out-of-Plane Displacement (OPD) near crack rips at $\Delta K=12$ and $18 \mathrm{MPa} \times \mathrm{m}^{1 / 2}$ in various testing conditions. The width of half monotonic plastic zone size $r_{p} / 2$ is indicated to compare with the OPD width.
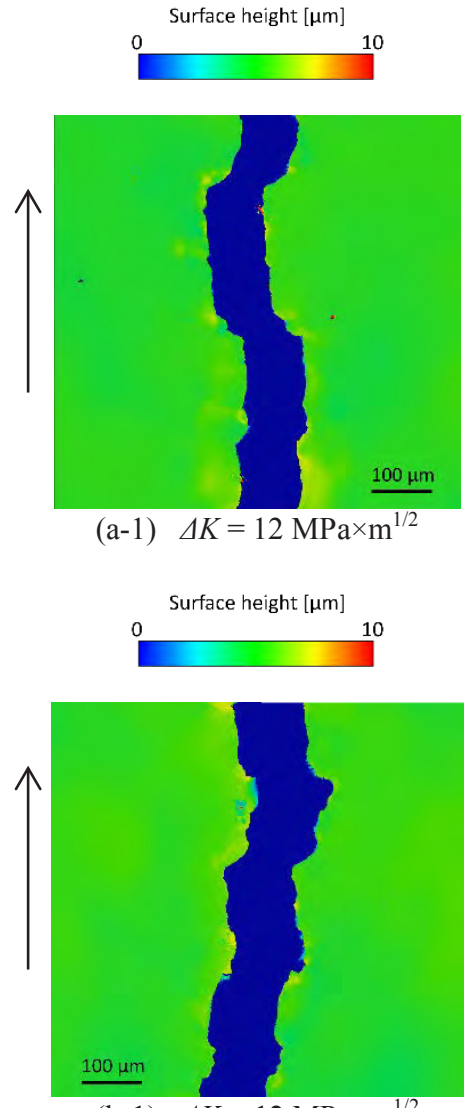

(b-1) $\Delta K=12 \mathrm{MPa} \times \mathrm{m}^{1 / 2}$ (a) In nitrogen, $f=20 \mathrm{~Hz}$
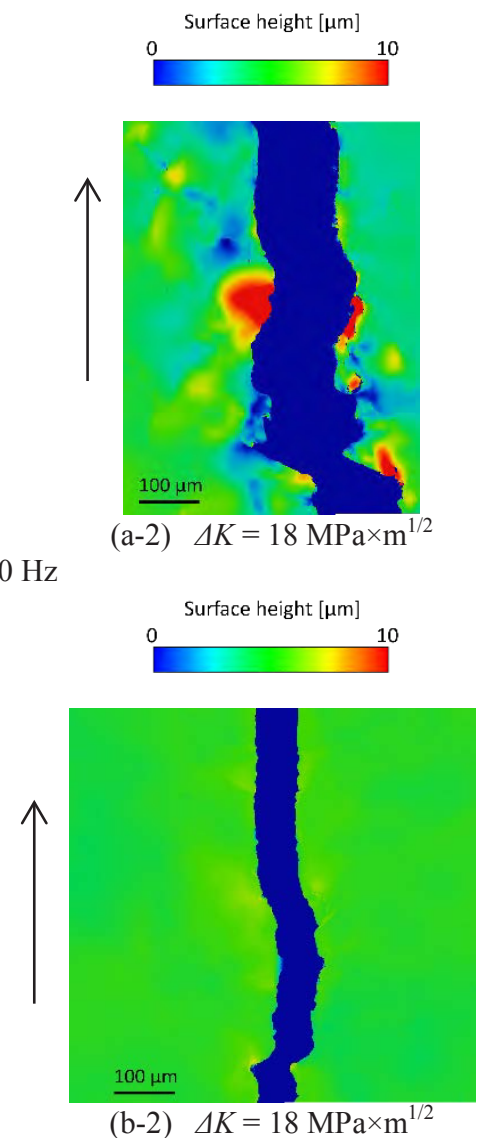

(b) In hydrogen, $f=20 \mathrm{~Hz}$

Fig. 6. High magnification images of surface topography near crack rips in nitrogen $(3.5 \mathrm{MPa}, f=20 \mathrm{~Hz})$ and in hydrogen $(35$ $\mathrm{MPa}, f=20 \mathrm{~Hz}$ ). The arrows indicate the direction of crack propagation. 
$\mathrm{MPa} \times \mathrm{m}^{1 / 2}$, the surface topographic image in hydrogen (Fig. 6 (b-2)) shows no significant surface unevenness as same as at $\Delta K=12 \mathrm{MPa} \times \mathrm{m}^{1 / 2}$, meanwhile a heterogeneous surface unevenness over a range of few hundreds of $\mu \mathrm{m}$ from the crack path is clearly observed in nitrogen (Fig. 6 (a-2)). According to Rice [11], the cyclic plastic zone size $\Delta r_{p}$ in plane stress condition can be estimated by following equation:

$$
\Delta r_{p}=\frac{1}{\pi}\left(\frac{\Delta K}{2 \sigma_{y}}\right)^{2}
$$

The value of half cyclic plastic zone size $\Delta r_{p} / 2$ is

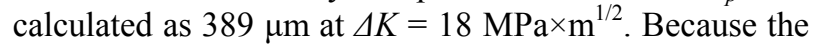
surface unevenness observed in Fig. 6 (a-2) spreads out within 100-200 $\mu \mathrm{m}$ distance from the crack path which is close to the calculated value of $\Delta r_{p} / 2$, the surface unevenness observed in Fig. 6 (a-2) is likely a result of cyclic plasticity in front of the crack tip. Therefore, from the results of OPD measurement, it can be concluded that the presence of hydrogen inhibits the cyclic plasticity at the crack tip in the accelerated regime, while hydrogen does not influence on the monotonic plasticity.

\section{Discussion}

\subsection{Fracture mechanism in non-accelerated regime}

From the obtained results of TEM observation in the section 3.1, it has been revealed that the dislocation cell structure is formed in the grains beneath intergranular facets in the non-accelerated regime in hydrogen. The presence of dislocation cell structure means that a certain amount of plastic strain is introduced to grains even though a macroscopic feature of fracture surface represents a brittle intergranular fracture. This introduced plastic strain in the grains has been also confirmed by the presence of slip markings on intergranular facets as mentioned in the section 3.1. A similar dislocation cell structure beneath intergranular fracture surface in hydrogen has also been confirmed in the tensile tests in a pure iron by Wang et al. [12] and in a pure nickel by Martin et al. [13]. The diameter of dislocation cells observed in their studies is $200-500$ $\mathrm{nm}$ which is slightly smaller than the one in this study. In addition, Nishikawa et al. [14] has performed an in-situ observation of intergranular crack propagation in a low carbon steel under gaseous hydrogen, and confirmed the formation of slip bands in grains in front of intergranular crack. This result also demonstrates the presence of plastic strain introduced near the crack tip of the intergranular crack in hydrogen.

In order to explain the mechanism of hydrogeninduced intergranular fracture, Wang et al. [12] proposed "a hydrogen-induced plasticity-mediated decohesion process". Their model is based on the interaction between dislocation-dragged hydrogen atoms and grain boundaries. In their model, the solute hydrogen atoms in the vicinity of crack tip promote a slip activity in the grains. The enhanced mobile dislocations in slip planes transport and redistribute the hydrogen atoms to grain boundaries. Consequently, the highly accumulated hydrogen atoms at grain boundaries weaken cohesive energy of grain boundaries by the HEDE mechanism, and result in a grain boundary decohesion.

\subsection{Fracture mechanism in accelerated regime}

Another noteworthy result of the TEM observation is that the observed dislocation structure in the accelerated regime in hydrogen was a randomly distributed dislocation tangles without any cell or vein structure. This type of dislocation structure implies that the fracture at the crack tip occurred before the plastic strain leached to the sufficient level to form the dislocation cell structure, hence the fracture mode in hydrogen became more brittle-like crack propagation with less plasticity at the crack tip compared with the one in air. This agrees well with the suppression of cyclic plasticity at a crack tip by hydrogen observed in the OPD measurement and the presence of brittle striation on the fracture surface in the accelerated regime.

A series of researches by Kyushu university has also investigated the dislocation structure in the vicinity of the fatigue crack tip in the accelerated regime in a pure iron [15], a low carbon steel [16] and a Fe-Si alloy[17-19]. In these studies, the one in a pure iron by Ogawa et al. [15] has to be especially noted here. They conducted a crack propagation test in a pure iron and performed a Grain Reference Orientation Deviation (GROD) analysis and a TEM observation of the crosssection beneath fracture surface in the accelerated regime (at $\Delta K=17 \mathrm{MPa} \times \mathrm{m}^{1 / 2}$ ). Their GROD analysis showed a great reduction of plastic strain distribution within few hundreds $\mu \mathrm{m}$ from a crack path in hydrogen, although there was a deviation of plastic strain distribution along a crack path showing a slightlydeformed part and an almost non-deformed part. Their TEM observations in the non-deformed part presented the dislocation structure similar to the one in this study, a scattered dislocation tangles distribution. On the other hand, the dislocation cell structure was observed within limited depth $(<2 \mu \mathrm{m})$ in a slightly-deformed part. Their obtained results of the GROD analysis and the TEM observation are in a good agreement with the ones of the OPD measurement and the TEM observation in this study, respectively.

It has to be mentioned that a clear modification of OPD around the crack path due to hydrogen has been observed in a 15-5PH steel by Bilotta et al. [20]. In a 15$5 \mathrm{PH}$ steel, it has been confirmed that hydrogen enhances maximum OPD of monotonic plastic zone without change in the OPD width, namely, increases the slope of OPD curve. This result is different from the one obtained in this study since no influence of hydrogen on the OPD of monotonic plastic zone is observed in Armco iron. However, the size of OPD of monotonic plastic zone in a $15-5 \mathrm{PH}$ is few hundreds $\mu \mathrm{m}$ which is the same order of size as the cyclic plastic zone in Armco iron clearly influenced by hydrogen. Therefore, this suggests that hydrogen affects only the plasticity within a certain 
distance from the crack tip. Another possible explanation is a difference in crack propagation mechanism. Bilotta et al. also confirmed that the hydrogen-enhanced FCG in a $15-5 \mathrm{PH}$ steel is governed by $K_{\max }$ rather than by $\Delta K$. By considering this fact, the influence of hydrogen on plasticity at crack tip and a mechanism of consequent FCG enhancement might be different between high strength steels like a $15-5 \mathrm{PH}$ steel and ductile steels or a pure iron.

Murakami et al. [21] and Matsuoka et al. [22] have proposed a model of FCG acceleration mechanism as called a "hydrogen-enhanced successive fatigue crack growth (HESFCG)" model based on a hydrogen-induced localization of plasticity. In this model, a steep gradient of hydrogen content causes a localization the plasticity at the crack tip and prevents a crack tip blunting. As a consequence, a crack tip becomes sharp and a crack advances continuously during a rising part of cyclic loading, resulting in the increase of crack advancement per one cycle and the difference in the fracture morphology. The experimental results obtained in this study basically agree with the HESFCG model. Based on the HESFCG model, it is elicited that an accelerated crack advancement does not exceed a maximum value of a Stretched Zone Width $\left(S Z W_{\max }\right)$ defined as: $S Z W_{\max }=$ $95\left(1-v^{2}\right)\left(K_{\max } / E\right)^{2}$ [22], where $v$ is Poisson's ratio and $E$ is an elastic modulus. In the case of Armco iron, $v$ is 0.3 and $E$ is $207 \mathrm{GPa}$, hence $S Z W_{\max }$ is calculated as $9.96 \times 10^{-7} \mathrm{~m}$ at $\Delta K=20 \mathrm{MPa} \times \mathrm{m}^{1 / 2}$. It can be confirmed in Fig. 1 that this value of $S Z W_{\max }$ is slightly lower but fairly close to the FCGR at $f=0.2 \mathrm{~Hz}$ in hydrogen of 35 $\mathrm{MPa}$. This fact predicts that this FCGR value already reaches the upper limit of acceleration.

\section{Conclusion}

In this study, a multi-scale analysis of the plasticity ahead of crack tip in hydrogen-affected fatigue crack propagation has been performed in a commercially pure iron, Armco iron. The main conclusions obtained in this study are shown below.

(1) In a non-accelerated regime, the dislocation structure developed immediately beneath fracture surface consists of a cell structure with the cell diameter of few $\mu \mathrm{m}$, identical to the one observed in air. This indicates that a certain amount of plastic strain is introduced into grains even in a brittle intergranular fracture. The plastic strain and slip activity in front of a crack tip may explain a mechanism of hydrogen-induced intergranular fracture.

(2) In an accelerated regime, the dislocation structure near the crack tip is a scattered dislocation tangles without any cell or vein structure.

(3) From the results of the OPD measurement, it has been clarified that hydrogen localizes cyclic plasticity ahead of a crack tip in an accelerated regime, on the other hand, hydrogen does not affect monotonic plasticity.

(4) Based on the experimental results mentioned in (2) and (3), the hydrogen-enhanced FCG in the accelerated regime can be understood by a model based on the localization of cyclic plasticity due to hydrogen.

\section{Acknowledgement}

Funding from the Poitou-Charentes region (G. Bilotta and T. Shinko $\mathrm{PhD}$ thesis grants) is gratefully acknowledged. This work pertains to the French Government program "Investissements d'Avenir" (LABEX INTERACTIFS, reference ANR-11-LABX0017-01).

\section{References}

1. G. Hénaff, G. Odemar, A. Tonneau-Morel, Int. J. Fatigue, 29, 1927-1940, (2007).

2. Y. Murakami, S. Matsuoka, Eng. Fract. Mech., 77, 1926-1940, (2010).

3. S.P. Lynch, Metall. Mater. Trans. A, 44, 12091229, (2012).

4. R.A. Oriani, P.H. Josephic, Scr. Metall., 6, 681688, (1972).

5. H.K. Birnbaum, P. Sofronis, Mater. Sci. Eng. A, 176, 191-202, (1994).

6. S.P. Lynch, N.E. Ryan, Proc. Second Int. Congr. Hydrog. Met., 369-376, (1977).

7. G. Bilotta, G. Hénaff, G. Benoit, C. Moriconi, D. Halm, M. Arzaghi, ASME 2015 Press. Vessel. Pip. Conf., 1-8, (2015).

8. G. Bilotta, M. Arzaghi, G. Hénaff, G. Benoit, D. Halm, Vol. 6B Mater. Fabr., V06BT06A026, (2016).

9. Z. Sun, C. Moriconi, G. Benoit, D. Halm, G. Henaff, Metall. Mater. Trans. A, 44, 1320-1330, (2012).

10. J. Schijve, Fatigue of Structures and Materials, Second edi, (2009).

11. J.R. Rice, Fatigue Crack Propag., ASTM STP 4, 247-309, (1967).

12. S. Wang, M.L. Martin, P. Sofronis, S. Ohnuki, N. Hashimoto, I.M. Robertson, Acta Mater., 69, 275-282, (2014).

13. M.L. Martin, B.P. Somerday, R.O. Ritchie, P. Sofronis, I.M. Robertson, Acta Mater., 60, 27392745, (2012).

14. H. Nishikawa, Y. Oda, H. Noguchi, J. Solid Mech. Mater. Eng., 5, 263-278, (2011).

15. Y. Ogawa, D. Birenis, H. Matsunaga, A. Thøgersen, Ø. Prytz, O. Takakuwa, J. Yamabe, Scr. Mater., 140, 13-17, (2017).

16. H. Nishikawa, Y. Oda, Y. Takahashi, H. Noguchi, J. Solid Mech. Mater. Eng., 5, 179190, (2011).

17. Y. Takahashi, M. Tanaka, K. Higashida, H. Noguchi, Scr. Mater., 61, 145-148, (2009).

18. Y. Takahashi, J. Sakamoto, M. Tanaka, K. Higashida, H. Noguchi, Scr. Mater., 64, 157160, (2011).

19. Y. Takahashi, J. Sakamoto, M. Tanaka, K. 
Higashida, H. Noguchi, Scr. Mater., 64, 721724, (2011).

20. G. Bilotta, G. Henaff, D. Halm, M. Arzaghi, Int. J. Hydrogen Energy, 42, 10568-10578, (2017).

21. Y. Murakami, T. Kanezaki, Y. Mine, 39, 13271339, (2008).

22. S. Matsuoka, N. Tsutsumi, Y. Murakami, Trans. Japan Soc. Mech. Eng. Ser. A (in Japanese), 74, 1528-1537, (2008). 